\title{
Nous pouvons faire de "sightseeing " ensemble: le contact linguistique au niveau lexical dans la production écrite en FLE des lycéens maltais
}

\author{
Anne-Marie Bezzina
}

Faculté des Sciences de l'Education, Université de Malte ${ }^{1}$

\begin{abstract}
Résumé. Cette étude analyse les erreurs lexicales dans la production écrite de lycéens maltais étudiant le français L2 aux niveaux B1 et B2, en se focalisant sur les erreurs attribuables à l'influence de la L1, qui, à Malte, comprend le maltais, l'anglais et l'italien. Des réflexions sont faites sur la difficulté d'admettre une idéologie translinguistique, tolérante de l'appui fourni par la L1 dans l'écriture en L2, dans le contexte d'un examen à un niveau avancé, avec ses normes de correction linguistique. Un corpus de copies d'examen aux niveaux Avancé et Intermédiaire est fouillé pour les possibilités de phénomènes de transfert, catégorisés en cinq types, émanant de difficultés orthographiques, de choix de mots, ou sémantiques, ces dernières provoquant l'utilisation des faux-amis. Les résultats sont comparés aux conclusions d'études faites dans les cadres maltais et international. La fréquence des contacts linguistiques dans le corpus est probablement attribuable tant à l'alternance codique, comportement omniprésent à Malte, qu'à la nature même de la rédaction en L2, activité forcément bilingue. Des calculs statistiques permettent des comparaisons des fréquences de contact aux niveaux Avancé et Intermédiaire, entre les copies mieux notées et les moins bien notées, comme entre les tâches plus exigeantes et les tâches plus simples.
\end{abstract}

\begin{abstract}
Nous pouvons faire de "sightseeing" ensemble: language contact on the lexical level in Maltese Sixth Form learners' writing in French L2. This study analyses lexical errors in French L2 writing tasks carried out by Maltese students studying French at B1 and B2 level, focusing on errors attributable to L1 influence, including, in the Maltese context, transfer from Maltese, English and even Italian. Reflections are made concerning the difficulty to adhere to a translingual ideology, tolerant of L1 support in L2 writing, in an advanced language examination setting with its norms and demands of accuracy. A corpus of Intermediate and Advanced Level examination scripts is searched for occurrences of possible transfer phenomena, which are categorized into five different types, stemming from orthographic uncertainty, knowledge gaps on the level of word choice, and difficulties regarding meaning, instigating a tendency to resort to false friends. Results are compared with findings in the local and international literature. The frequency of language contact phenomena is probably attributable both to the ever-present codeswitching behaviour in the Maltese bilingual context, as well as to the nature of L2 writing, in itself a bilingual activity. Basic statistical calculations allow comparisons and interpretations of frequencies of contact between the Advanced and Intermediate sub-corpora, between higher and lower proficiency levels, as well as between more and less demanding tasks.
\end{abstract}

\footnotetext{
${ }^{1}$ anne-marie.bezzina@um.edu.mt
} 


\section{Le contexte maltais : une brève description}

A $93 \mathrm{~km}$ au sud de la Sicile, les îles maltaises constituent le plus petit Etat de l'Union Européenne, avec une population d'à peu près 500,000 habitants. L'immigration multiethnique est en train de causer une expansion rapide de la communauté. Le maltais, langue basée sur l'arabe mais ayant à travers les siècles intégré de fortes influences siciliennes, italiennes et anglaises, est la langue nationale de Malte. Le maltais et l'anglais sont les deux langues officielles des îles. Beaucoup de Maltais ont aussi une certaine connaissance de l'italien. Pourtant, les jeunes générations, qui préfèrent les médias en maltais et en anglais, sont de manière générale bilingues, mais les nombreux habitants de nationalité étrangère ont rendu la palette linguistique des îles bien colorée.

\subsection{Un aperçu de la situation sociolinguistique de Malte}

Le maltais est de loin la langue principalement utilisée par les habitants de nationalité maltaise pour la communication quotidienne. Dans la fonction publique, le maltais jouit d'un statut institutionnel très fort. En revanche, dans le secteur privé, la formalité, surtout à l'écrit, tend à impliquer le choix automatique de l'anglais. Une minorité de la population autochtone, et notamment une partie de l'élite, parlent anglais comme L1. La langue privilégiée du système éducatif est l'anglais. De plus en plus d'enfants des classes moyennes sont locuteurs d'anglais L1, sous l'effet des ambitions sociales de leurs parents, et parce qu'ils sont très en contact avec les moyens technologiques en langue anglaise.

En fait, en parlant 'en maltais', on alterne les deux codes de manière presque généralisée. Ceci est souvent tout simplement le fruit de l'habitude, mais a parfois lieu à cause du prestige associé à l'anglais, lorsque les locuteurs veulent afficher leur niveau d'éducation et / ou leur statut social.

\subsection{L’apprentissage des langues à Malte}

Le bilinguisme est un des piliers de l'éducation à Malte. Les enfants apprennent le maltais et l'anglais dès le début du cycle primaire. Les manuels et les examens de la plupart des matières sont en anglais, mais l'alternance codique caractérise l'interaction en classe.

Les élèves doivent obligatoirement choisir et étudier une langue étrangère durant leur cycle secondaire qui dure cinq ans. Le français peut être choisi en tant que langue étrangère et les apprenants n'ont généralement pas de contact avec la langue en dehors des classes. A la fin du collège, les élèves passent des examens nationaux au niveau appelé Secondary Education Certificate (SEC), organisés par le bureau MATSEC, une branche de l'Université de Malte dédiée à l'évaluation. Pour les langues étrangères, ces examens correspondent au niveau A2+ ou B1 précoce.

Au niveau du lycée, qui dure deux ans et auquel s'intéresse cette étude, il faut choisir deux matières au niveau Avancé et trois au niveau Intermédiaire, pour pouvoir ensuite accéder aux cursus universitaires. A la fin du lycée, il faut réussir les examens de ces matières, eux aussi organisés par le bureau MATSEC. Quant aux langues étrangères, le niveau Avancé correspond au niveau B2 et le niveau Intermédiaire au niveau B1. 


\subsection{Format des examens de français aux niveaux Avancé et Intermédiaire}

L'examen de français au niveau Avancé est noté sur un total de 400 points répartis sur 4 épreuves. Dans cette étude, nous portons notre attention sur les deux exercices dans lesquels les lycéens doivent librement écrire un texte plutôt long en français, à savoir l'Analyse d'un texte non littéraire, notée sur 60 points, et la Rédaction, notée sur 100 points, pour laquelle les élèves doivent écrire entre 350 et 400 mots.

L'examen au niveau Intermédiaire est noté sur 100 points. Dans cette étude l'attention se porte sur la Rédaction ( 25 points), pour laquelle il faut écrire entre 230 et 250 mots.

\section{0 Études sur le contact de langues dans le contexte éducatif maltais}

Plusieurs études ont porté sur le contact de langues dans les échanges verbaux qui ont lieu dans les classes maltaises. Une partie de la recherche s'est focalisée sur la communication verbale en classe de L2 (Caruana \& Camilleri Grima, 2014 ; Camilleri Grima \& Caruana, 2016; Gauci \& Camilleri Grima, 2013 pour l'italien ; Bezzina, 2016 et 2017 ; Bezzina \& Gauci, 2018 pour le français). Certaines montrent que la langue cible est loin d'être la langue dominante en classe (Bezzina \& Gauci, 2018), et que l'alternance entre le maltais, langue de la communication générale, l'anglais, langue du métalangage, et la langue cible, utilisée pour se référer au contenu, est le véhicule de communication verbale le plus courant. Ceci est facile à comprendre lorsqu'on considère la classe de langue étrangère comme un cadre naturel de contact linguistique, d'autant que ces classes se situent dans le contexte maltais où l'alternance codique est un comportement " inné » des locuteurs. Les corpus des études mentionnées ci-dessus illustrent ce va-et-vient spontané entre le maltais, l'anglais et la langue cible. Bien que l'on zigzague de façon continue, le mouvement ne crée pas de complexité pour le décodage ; il facilite plutôt la compréhension grâce au recours aux mots connus des élèves.

En général, les chercheurs mentionnés ci-dessus concluent que l'alternance verbale sert d'atout, plutôt que de déficit ou de source d'interférence. Ses fonctions positives sont soulignées, telles que sa contribution dans la création d'un environnement plus décontracté, sa façon d'accroître la motivation et la compréhension, le fait qu'elle favorise des essais de la part des élèves lorsqu'ils tentent des approximations vers la langue cible, et son efficacité dans la gestion de la classe (Camilleri Grima \& Caruana 2016, Bezzina 2017, Bezzina \& Gauci 2018). L'alternance codique permet aux bilingues de puiser dans leurs multiples ressources linguistiques pour s'exprimer; par la suite, les élèves sont plus en mesure de participer dans l'interaction sans se sentir découragés ou perdus comme cela pourrait être le cas dans une classe où il faut exclusivement employer la L2. En même temps, pourtant, les chercheurs préconisent davantage de pratique orale de la L2, qui est trop peu présente dans les interactions en classe (Bezzina 2017, Bezzina \& Gauci 2018).

\subsection{L'alternance codique et la production écrite en langue étrangère}

La question qui surgit naturellement à ce point est si cette évaluation positive de l'alternance codique dans l'interaction verbale s'étend au mélange de codes dans la production écrite des élèves, dans le cadre de l'apprentissage des langues étrangères. Certes, un nombre de chercheurs sont d'avis que la L1 profite au processus complexe de l'écriture en L2 en agissant d'outil de résolution de problèmes étayant cette tâche difficile 
(Knutson, 2006). Friedlander (1990) croit que les apprenants écrivant en L2 devraient être encouragés à utiliser la L1 en produisant les premières ébauches de leur texte. Qahfarokhi \& Biria (2012) accentuent leur conviction que les enseignants devraient former les élèves à tirer avantage de leurs ressources bilingues en encourageant davantage d'alternance; d'après eux, le recours à la L1 peut améliorer la qualité des textes produits, surtout dans le cas des élèves de niveau intermédiaire. Leurs données montrent que les élèves moins doués en L2 alternent vers la L1 beaucoup plus souvent, et qu'une fréquence élevée d'alternance semble faciliter le processus de rédaction quand la difficulté de la tâche est importante. Ayant obtenu un résultat similaire dans son étude de cas impliquant une apprenante chinoise très douée en anglais L2, Qi (1998) note une augmentation systématique d'emploi de la L1 lorsque les demandes cognitives des tâches d'écriture sont plus exigeantes. Pour Knutson (2006), sa deuxième participante plus douée en écriture en français L2 fait largement emploi de la traduction mentale, ce qu'elle interprète comme signe que la réflexion en L1 peut être efficace pendant l'écriture en L2.

Plusieurs chercheurs attestent que la réflexion en L1 appuie les sous-catégories composant le processus de rédaction, tant au niveau micro (par exemple, dans la recherche $\mathrm{du}$ vocabulaire), qu'au niveau de l'organisation discursive : par exemple, dans la planification de la structure globale du texte, les réflexions sur le genre, la révision du texte déjà écrit, ou encore la réflexion sur l'adéquation de mots particuliers par rapport au sens que le participant souhaite véhiculer (Knutson, 2006 ; van Weijen \& al., 2009). De même, la participante de l'étude de cas de Qi (1998) a souvent pu accéder à une idée en l'encodant d'abord dans la L1 pour ensuite la développer. Cette phase de développement paraît caractérisée par une alternance fréquente, qui facilite l'expression des idées individuelles. En plus, l'alternance aide à la vérification du sens des items lexicaux : le choix et le sens d'un mot difficile en L2 sont vérifiés par la rédactrice en se référant à la traduction correspondante en L1. L'alternance rapide et automatique aide à éviter la surcharge de la mémoire lorsque des tâches complexes doivent être abordées (Qi, 1998).

Pourtant, alors que la recherche mentionnée ci-dessus considère le mélange de langues comme une aide utile au processus bilingue de l'écriture en L2, elle tend à ne pas montrer la qualité du produit fini, ou à ne pas s'y attarder. Elle semble mettre de côté la question des critères d'évaluation de la production écrite en L2. La question se présente donc de la façon dont les instances de mélange linguistique doivent être perçues dans l'évaluation formelle, surtout lorsque les apprenants ont atteint un certain niveau de spécialisation dans la langue, et que leur écrit est parsemé de mots, et / ou de structures trahissant l'influence de la L1.

$\mathrm{Au}$ bout du compte, c'est le résultat final, le texte dépersonnalisé et détaché du processus de rédaction qui est lu et évalué dans des contextes formels tels que ceux du corpus de la présente étude. Ecrire dans un scénario de classe ou d'examen avec l'objectif de maîtriser la communication en L2 s'inscrit dans un cadre formel. Contrairement à la communication qui se fait dans des cadres informels, les contextes d'écriture formelle nécessitent normalement la séparation des langues (Busuttil Bezzina, 2013). C'est au vu de ce genre de préoccupations que Gevers (2018) accentue les demandes distinctes de la communication orale et écrite. Il reconnaît l'orientation des premiers promoteurs du transcodage en tant que proposition nécessaire pour considérer les variétés non standard d'anglais des apprenants comme ressources qui pourraient servir à résister les forces marginalisantes de l'anglais écrit standard (Gevers 2018), mais souligne la nécessité que les enseignants tiennent compte des traits qui distinguent la langue écrite de la langue orale lorsqu'ils évaluent la production écrite des apprenants. Gilyard (2016) le précède en exprimant le souci que le translinguisme risque de gommer les différences interlinguistiques. Il est donc clair que le translinguisme est encore aux prises avec la 
question de l'évaluation linguistique, et avec l'effet des normes attendues et largement partagées, qui se reflètent dans les buts derrière les pratiques et l'enseignement de l'écriture (Gevers, 2018 ; Gilyard, 2016 ; Lee, 2016).

Notre corpus consiste de copies d'examen rédigées aux niveaux Avancé et Intermédiaire par des candidats visant à obtenir un bon résultat qui leur permettra d'être admis aux cursus de l'Université de Malte ; leur performance en langue cible se doit donc d'être convaincante. Dans ce contexte, la correction grammaticale se voit attribuer beaucoup d'importance. On évalue de près l'emploi du vocabulaire approprié, de la précision des termes et expressions qui doivent être variés et aller au-delà de l'élémentaire, étant donné qu'une partie de la note finale est spécifiquement réservée au vocabulaire. On ne s'attend pas à ce que les examinateurs se montrent indulgents vis-à-vis des mots et phénomènes morphosyntaxiques calqués sur leurs équivalents en L1, voire empruntés tels quels à la L1. Ceci est peut-être l'une des raisons pourquoi, dans le contexte du système éducationnel maltais fortement basé sur les examens, les analyses des erreurs ont été privilégiées par opposition aux expérimentations visant à comprendre le processus de rédaction bilingue, qui seraient plus ou moins tolérantes de l'influence de la L1.

\subsection{Les études sur le contact des langues à l'écrit}

Les études portant scientifiquement sur le contact des langues à l'écrit en contexte maltais sont assez rares. Certaines visent les différents schémas de contact qui émergent dans des contextes formels, par opposition aux contextes informels. Dans le chat électronique, l'alternance codique et des formes spéciales d'écriture telles que celles décrites dans Caruana \& Brincat (2011) et Busuttil Bezzina (2013) sont de règle et aident les participants à s'exprimer avec les effets désirés et de façon décontractée. D'autre part, on s'attend à la séparation des langues dans les contextes d'écriture formelle, et les individus cherchent à respecter cette norme partagée dans leurs écrits (Busuttil Bezzina, 2013).

Quelques études ont décrit le contact linguistique dans la production écrite des apprenants de langues étrangères. Seychell (1996) fait une analyse des erreurs à partir d'un corpus de rédaction libre effectuée par des étudiants de FLE au niveau Avancé, en étudiant les interférences provoquées par l'anglais et l'italien, mais en concluant hâtivement que « [d] ans le cas des apprenants maltais c'est l'anglais et l'italien, langues intermédiaires, non pas le maltais qui perturbent l'apprentissage du français à tous les différents niveaux de l'organisation du langage » (1996: 13). En revanche, Sammut (2017) effectue une analyse des erreurs syntaxiques à partir de rédactions d'apprenants de FLE (niveau A2) écrites hors du contexte des examens, et identifie des cas de contact avec tant le maltais que l'anglais.

Caruana (2011) étudie le contact des langues, à savoir l'influence du maltais et de l'anglais en tant que L1, sur l'écrit des candidats de l'examen SEC d'italien L2. Il a trouvé de nombreux cas de contact dans la production écrite 'en italien', et atteste que l'influence provient surtout du maltais, notamment parce que la plupart des mots maltais sont dérivés d'items lexicaux italiens. Caruana trouve que les correspondances entre graphèmes maltais et italiens pour des phonèmes identiques ou peu différents, donnent lieu à de fréquentes erreurs d'orthographe sous l'influence des graphèmes maltais (par exemple, *xjenzato pour l'italien scienzato (scientist), à partir du mot maltais xjenzat. Il analyse aussi l'influence au niveau lexical, et suggère que le transfert du maltais et de l'anglais sur l'italien est moins évident, mais toujours présent, au niveau morphosyntaxique. 


\subsection{Problématique et méthodes de recherche}

La présente étude adopte une approche similaire à celle de Caruana (2011) dans la mesure où elle vise à identifier des cas de contact entre le maltais et l'anglais L1 et le français L2 dans des copies d'examen. Pourtant, nous nous limitons ici à étudier le contact lexical, et des conclusions différentes sont attendues : le corpus de la présente étude se compose de copies d'examens de jeunes âgés de 18 ans, qui ont fait deux autres années d'étude en langue cible. Du coup, il se peut qu'ils aient davantage surmonté le problème de l'influence de leur L1 dans leur production écrite. Une autre question à laquelle il faut répondre est si l'influence du maltais est plus forte que celle de l'anglais sur les textes français, comme c'est le cas dans le corpus d'italien, et si elle donnerait lieu à de fréquentes erreurs d'orthographe. Le maltais contient très peu de mots dérivés du français, à la différence de son importante source lexicale italienne.

La présente étude se focalisant sur le contact linguistique dans la production écrite aux niveaux Avancé et Intermédiaire de FLE, le corpus se compose de parties spécifiques des copies des examens passés en 2018 à ces deux niveaux. Le corpus nous a été rendu disponible par le bureau MATSEC. Il a été assuré que nous n'ayons accès ni aux noms des candidats, ni à d'autres détails qui pourraient identifier de petits groupes de candidats.

Dans les échantillons que nous avons analysés à chaque niveau et pour chaque exercice, un effort a été fait pour que la gamme des copies soit aussi équilibrée que possible, avec un mélange de copies ayant reçu des notes élevées, moyennes, et basses. Le Tableau 1 indique le nombre de copies retenues à partir de chaque lot, et la gamme des notes dans chaque cas, après que les notes ont été proportionnellement recalculées sur 100 quand elles ne l'étaient pas déjà. Les calculs des notes sur 100 étaient nécessaires pour permettre des comparaisons. La gamme des notes des rédactions au niveau Avancé s'étend sur les deux sessions, avec les copies des meilleures notes retenues à partir de la session de mai et les copies avec des notes vers le bas de la gamme retenues à partir de la session 'de rattrapage' de septembre. La note obtenue dans tel ou tel exercice a été considérée comme reflet de la qualité du texte et du degré de compétence linguistique de chaque candidat.

Tableau 1 : Taille, type et gamme des notes des échantillons de copies retenues pour l'analyse

\begin{tabular}{|c|c|c|c|c|}
\hline $\begin{array}{c}\text { Nombre de } \\
\text { copies }\end{array}$ & Niveau & $\begin{array}{c}\text { Type } \\
\text { d'exercice }\end{array}$ & $\begin{array}{c}\text { Session (mai - } \\
\text { session principale / } \\
\text { septembre - session } \\
\text { 'de rattrapage') }\end{array}$ & Gamme des notes \\
\hline 12 & Intermédiaire & Rédaction & mai & $\begin{array}{l}\text { de la plus haute } 96 / 100 \\
\text { à la plus basse } 12 / 100\end{array}$ \\
\hline 6 & Avancé & Rédaction & mai & $\begin{array}{l}\text { de la plus haute } 81 / 100 \\
\text { à la note moyenne } \\
62 / 100\end{array}$ \\
\hline toutes les 9 & Avancé & Rédaction & septembre & $\begin{array}{l}\text { de la note moyenne } \\
66 / 100 \text { à la plus basse } \\
32 / 100\end{array}$ \\
\hline 9 & Avancé & $\begin{array}{c}\text { Analyse d'un } \\
\text { texte non } \\
\text { littéraire }\end{array}$ & mai & $\begin{array}{l}\text { de la plus haute } 83 / 100 \\
\text { à la plus basse } 35 / 100\end{array}$ \\
\hline
\end{tabular}

Ces échantillons ont été soumis à une recherche manuelle d'occurrences de contacts de langues. Le contact est dans cette étude décrit par moyen des termes "transfert», « influence» ou « interférence», sans qu'aucun de ces termes ne se distingue par une connotation forcément positive ou négative. Le critère à partir duquel il a été décidé de la 
présence d'une occurrence de contact a été la possibilité qu'une structure ou un mot aient été utilisés sous l'influence d'une autre langue connue : une occurrence de contact survient donc chaque fois qu'un segment contient une structure ou un mot considérés comme résultats d'un transfert potentiel provenant de la L1. La définition retenue dans cette étude de « occurrence de contact linguistique » repose donc effectivement sur la possibilité qu'un emploi d'un item lexical ou d'une structure ait été produit sous l'effet d'une autre langue. En effet, dans beaucoup de cas, la cause d'un emploi particulier ne peut pas être définie avec certitude. Des solutions potentielles pour surmonter cette faiblesse méthodologique seront discutées plus loin. Il suffit pour l'instant de noter que le travail sur corpus a déterminé la nature de l'étude, qui se présente fondamentalement comme une analyse descriptive des erreurs.

Tous les cas individuels de contact potentiel ont été insérés, au sein des phrases entières dans lesquelles ils ont paru, dans des tableaux comportant plusieurs colonnes, dont l'une réservée à la description du type de transfert. Ceci a servi de base à la description qualitative des occurrences de contact. Bien que nous ayons relevé de nombreux cas de contact au niveau morphosyntaxique, pour des raisons d'espace nous nous focalisons dans la présente étude sur le contact au niveau lexical.

Des calculs statistiques du nombre d'occurrences impliquant tous les types de contact ont été effectués proportionnellement sur 100 mots pour chaque copie. Ceci a permis des comparaisons quantitatives entre différentes catégories du corpus.

\subsection{Nature linguistique des occurrences de contact linguistique au niveau lexical}

Plusieurs types de transfert potentiel de mots et d'expressions de la L1 ont été identifiés dans les copies, et sont énumérés et illustrés ci-dessous :

\subsection{Transferts dû à l'influence orthographique}

Cette catégorie de transferts comprend tant les mots qui sont écrits comme les mots de la L1 (qui dans ce travail se réfère au maltais, à l'anglais et parfois à l'italien), que les mots intégrant un trait de l'orthographe du mot correspondant de la L1 :

a. L'auteur veut embellir son texte en utilisant la personification «battre le cœur de l'Afrique » (Av. Analyse 47, de l'anglais personification / du maltais personifikazzjoni, ces mots ayant seulement un 'n' dans les deux langues)

b. Les adjectif contribuent aussi à ce sense de positivité: "moderne, conquérante, décomplexée et apaisée » (Av. Analyse 7, de l'anglais sense)

c. Je te recommende de manger plus, mais ne mange pas trop... (Niveau Intermédiaire (Int.) Rédaction 4016, de l'anglais recommend)

d. Par example... (Av. Analyse 4, de l'anglais example)

e. ... des valuers différentes... (Av. Rédaction de septembre 7, de l'anglais values)

f. Chaque personne a un besoign de avoir un ami... (Av. Rédaction de septembre 8, de l'italien bisogno)

g. Ce type de texte pourrait etre trouver dans la rubrique voyage ou tourisme et donc utilise un language simple. (Av. Analyse 47, de l'anglais language) 


\subsection{La « francisation » d'emprunts lexicaux}

Un nombre d'items lexicaux paraissent être les résultats de mots de la L1 dotés de graphèmes français. D'autres semblent être les résultats d'inventions issues de processus d'analogie avec d'autres langues, le plus souvent le maltais et l'anglais. Dans ces cas le contact de langues mène à la création de formes hybrides, comme dans ces exemples :

a. ... la technologie a ses disavantage. (Av. Rédaction septembre 2, de l'anglais disadvatage)

b. D'abord, dans notre pays il y a beaucoup de travailles interessant et a une pague bonne... (Av. Rédaction septembre 4, du maltais / Italian paga; pour un bon salaire)

c. Les livres peuvent être un mode de rilassement... (Av. Rédaction septembre 7, du maltais rilassament / de l'italien rilassamento)

d. L'ordinateur est très popolaire avec l'adolescent parce qu'il aiment le liberté... (Int. Rédaction 4053, du maltais popolari / de l'italien popolare)

e. ... l'été est trés chaude, c'est perfecte pour nager. (Int. Rédaction 4045, de l'anglais perfect)

\subsection{Items lexicaux de la L1 utilisés tels quels}

Certains mots ont tout simplement été empruntés à d'autres langues et utilisées dans leur forme originelle. Ils sont fréquents au niveau Intermédiaire, mais presque tout à fait évités au niveau Avancé. Certains candidats en produisent parfois une quantité remarquable. Il est clair qu'il y a chez ces candidats une conscience que ce genre de comportement linguistique est inacceptable ; certains mots anglais sont insérés au crayon dans le texte français écrit au stylo bleu, ou sont mis entre guillemets. Cette catégorie se compose surtout de substantifs, mais de temps en temps on trouve dans le corpus un verbe ou un adjectif laissés tels que dans la L1, comme c'est le cas dans ces exemples :

a. Une advantage de l'ordinateur c'est que on peut faire plus de chose... (Int Essay 4053, anglais pour avantage)

b. ... l'ordinateur et le télévision sont actifs et attract l'attention des audiences différentes des beaucoup âgées. (Int Essay 4050, anglais pour attirent)

c. Nous pouvons faire de "sightseeing » ensemble (Int Essay 4045, anglais pour $d u$ tourisme)

d. Sur l'ordinateur, est très simple; un screen et keyboard, mais il rassurer que tout donnez-vous les informations, reaserch, films que vous étés trouvez. (Int Essay 4013, anglais pour écran, clavier, recherche)

e. Aussi, l'ordinateur on est utilise pour, musique, projets de l'ecole, pour online shopping, et beaucoup facilitalments. (Int Essay 4013, anglais pour shopping en ligne)

f. ... sur le television, l'individual est (able to $<$ au crayon $>$ ) regardée les programs, mais sur l'ordinateur, il y a beaucoup (things $<$ au crayon $>$ ) pour faire. (Int Essay 4013, anglais pour l'individu, peut, émissions, choses)

g. ... je le trouve difficile de parler a quelqu'un si non sur l'instant message. (Sep Adv Essay 2, anglais pour message instantané)

\subsection{Faux-amis}

Les exemples ci-dessous contiennent des items lexicaux qui existent bien en français; cependant, l'influence de leur valeur sémantique dans d'autres langues fait qu'ils finissent par être utilisés avec une acception différente du sens qu'ils expriment en français. 
a. Toutes les livres ont quelqu'aspect pour enseignent nous et nous pouvons étudier la theorie des sujets. (Av. Rédaction septembre 7, faux-ami de l'anglais subjects / maltais suggetti, pour matières)

b. Au cause des phrases longes et extensives il utilise plusieurs virgules pour aider la fluidité du texte. (Av. Analyse 7, faux-ami de l'anglais extensive, pour longues / élaborées (extensives s'utilise normalement en français dans les contextes de la chimie ou de l'agriculture)

c. Ce jour-là, une journée fascinante, dont le sol évantaille ses rayons brillants sur le monde... (Av. Rédaction mai 52, faux-ami de l'italien sole, pour soleil)

d. En plus, des jeunes décident d'arrêter le collège et obtenir un profession pour gagner d'argent. (Av. Rédaction septembre 9, faux-ami de l'anglais college, pour lycée)

e. Quant au champ lexical, la choix de vocabulaire est sensible car le texte souhaite sensibiliser le lecteur à la culture de Sénégale. (Av. Analyse 52, faux-ami de l'anglais sensible, pour adéquat / efficace)

f. Evidemment, nous trouvons des phrases concernant la géographie comme « occidentale », " Sahara », « la Méditerranée »... (Av. Analyse 52, faux-ami de l'anglais phrases, pour mots et expressions)

\subsection{Idiomatismes et expressions figées}

Les tentatives de produire des expressions figées paraissent souvent aboutir à des approximations, conséquence d'une traduction littérale à partir de la langue source. Certaines semblent être des versions allongées de faux-amis. Dans la plupart des cas, comme dans les exemples suivants, les expressions sont « francisées », ce qui reflète sans doute un effort de les rendre plus acceptables :

a. Quand il vient de communication sociale, la technologie la affecté (Av. Rédaction septembre 2, de l'anglais When it comes to, pour : Quant à)

b. L'Etat peut rendre amies avec des entreprises entrangéres d'autre pays qui ont des marques très connues. (Av. Rédaction septembre 3, de l'anglais make friends, pour : établir une collaboration avec)

c. $<$ début de nouveau paragraphe $>$ Sur un autre côté, quelques étudiants n'avaient pas le choix de ne pas travailler à cause de problèmes financiers. (Av. Rédaction septembre 6,

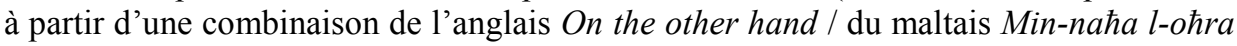
lit. De l'autre côté, pour : D'un autre côté)

d. En addition, un échange n'est pas un aprendissage pour les étudiants, mais aussi pour les familles qui doivent récevoir et acueillir un autre étudiant chez elles. (Av. Rédaction mai 4, de l'anglais In addition, pour : En plus / D'ailleurs)

e. Sur l'autre main sur le télé il y a de stations different que on peut regarder. (Int. Rédaction 4053, de l'anglais On the other hand, pour: D'un autre côté)

f. Mais tu m'inquiètes un peu pour être honnête. (Int. Rédaction 4016, de l'anglais to be honest, pour : à vrai dire)

g. ... l'ordinateur, est aide les personnes avec un disabilité, grâce a nouveu technologie et les personnes que comprends dans la technologie. (Int. Rédaction 4013, traduction littérale du maltais in-nies li jifhmu fit-teknologija)

\subsection{Indications émanant de calculs statistiques initiaux}

Afin de pouvoir comparer différentes composantes du corpus, un dénominateur commun a été utilisé en calculant, pour chaque rédaction ou analyse d'un texte non littéraire, le nombre proportionnel d'occurrences de contact linguistique sur 100 mots, même si les rédactions sont en fait bien plus longues. Le nombre réel d'occurrences de contact est donc 
plus grand que celui qui figure dans les tableaux ci-dessous. Dans ces considérations statistiques très simples, toutes les occurrences de contact, non seulement celles au niveau lexical, sont prises en compte. Le corpus abonde effectivement en cas de transferts aux niveaux morphologique et syntaxique, que nous décrirons dans une autre étude.

\subsection{La fréquence des occurrences de contact dans les rédactions du niveau Intermédiaire par rapport à celles du niveau Avancé}

Tableau 2. Nombre d'occurrences de contact dans les rédactions aux niveaux Intermédiaire et Avancé

\begin{tabular}{|l|l|}
\hline $\begin{array}{l}\text { Rédactions au niveau Intermédiaire - mai } \\
2018\end{array}$ & $\begin{array}{l}\text { Nombre d'occurrences de contact en calculant } \\
100 \text { mots par rédaction }\end{array}$ \\
\hline $\begin{array}{l}\text { 12 Rédactions (la plus large gamme de } \\
\text { compétences) }\end{array}$ & $\begin{array}{l}\mathbf{5 7 . 7} \text { occurrences (dans 12 rédactions x 100 } \\
\text { mots) }\end{array}$ \\
\hline $\begin{array}{l}\text { Rédactions au niveau Avancé - mai et } \\
\text { septembre }\end{array}$ & $\begin{array}{l}\text { Nombre d'occurrences de contact en calculant } \\
100 \text { mots par rédaction }\end{array}$ \\
\hline $\begin{array}{l}\text { 12 Rédactions (la plus large gamme de } \\
\text { compétences) }\end{array}$ & $\begin{array}{l}\mathbf{3 5 . 1} \text { occurrences (dans 12 rédactions x 100 } \\
\text { mots) }\end{array}$ \\
\hline
\end{tabular}

Le Tableau 2 illustre la quantité d'occurrences de contact de langues dans des rédactions aux niveaux Intermédiaire et Avancé quand chaque rédaction est supposée se composer de 100 mots. Dans les rédactions au niveau Avancé, il y a une fréquence bien inférieure d'occurrences de contact que dans les rédactions au niveau Intermédiaire.

\subsection{La fréquence des occurrences de contact dans les rédactions de candidats ayant des compétences supérieures par rapport à celles des candidats moins forts en L2}

Tant dans les rédactions au niveau Intermédiaire que dans celles au niveau Avancé des sessions de mai et de septembre, une tendance se dégage : en général, les rédactions mieux notées contiennent moins d'occurrences de transfert que les rédactions ayant reçu des notes inférieures. Le Tableau 3 montre le décalage numérique entre les quantités d'occurrences de contact dans les rédactions bien notées et les écrits moins bien notés.

Tableau 3. Nombre d'occurrences de contact dans les rédactions les mieux notées et dans les rédactions moins bien notées

\begin{tabular}{|l|l|}
\hline $\begin{array}{l}\text { Rédactions au niveau Intermédiaire - mai } \\
2018\end{array}$ & $\begin{array}{l}\text { Nombre d'occurrences de contact en calculant } \\
100 \text { mots par rédaction }\end{array}$ \\
\hline Les 6 rédactions les mieux notées & $\mathbf{1 9 . 6}$ occurrences \\
\hline Les 6 rédactions les moins bien notées & $\mathbf{3 4 . 1}$ occurrences \\
\hline Rédactions au niveau Avancé - mai et & $\begin{array}{l}\text { Nombre d'occurrences de contact en calculant } \\
100 \text { mots par rédaction }\end{array}$ \\
\hline septembre & $\mathbf{1 7 . 4 ~ o c c u r r e n c e s ~}$ \\
\hline Les 7 rédactions les mieux notées & $\mathbf{2 8 . 2}$ occurrences \\
\hline Les 7 rédactions les moins bien notées
\end{tabular}

En fait, tous les écrits des candidats les plus compétents ne contiennent pas systématiquement moins d'occurrences de contact, et l'inverse est également vrai. Les tendances dépendent largement des connaissances de chaque candidat en matière de vocabulaire, et en matière de règles morphosyntaxiques de la langue cible. Par exemple, au sein des six rédactions les mieux notées du niveau Intermédiaire, le nombre d'occurrences de contact sur 100 mots varie de 0 à 6,6 , et au sein des rédactions les moins bien notées, il 
varie de 9,5 à 2,2. De même, parmi les sept rédactions les mieux notées du niveau Avancé, le nombre d'occurrences sur 100 mots va de 1,4 à 3,4, et parmi les rédactions les moins bien notées, il va de 2,0 à 6,3 . Néanmoins, il ressort que globalement les rédactions les moins bien notées contiennent davantage d'occurrences de contact que les écrits mieux notés, à tous les deux niveaux.

\subsection{La fréquence des occurrences de contact dans la rédaction technique au niveau Avancé (Analyse d'un texte non littéraire)}

La tendance remarquée ci-dessus s'inverse dans le cas de l'Analyse du texte non littéraire, en ce sens que ce sont les écrits les mieux notés qui contiennent davantage d'occurrences de contact de langues, par rapport aux Analyses de texte moins bien notées. Il faut cependant traiter ces résultats avec prudence, à cause de la taille réduite de l'échantillon, qui se compose de neuf Analyses de texte, dont nous comparons les quatre les mieux notées et les quatre les moins bien notées. Le Tableau 4 indique le nombre d'occurrences de contact dans les Analyses de texte les mieux notées et dans celles ayant obtenu le moins de points.

Tableau 4. Nombre d'occurrences de contact dans les Analyses de texte les mieux notées et dans les moins bien notées (Niveau Avancé)

\begin{tabular}{|l|l|}
\hline Analyse d'un texte non littéraire - mai & Nombre d'occurrences de contact en calculant \\
& 100 mots par analyse \\
\hline Les 4 textes les mieux notés & $\mathbf{5 3}$ occurrences \\
\hline Analyse d'un texte non littéraire - mai & Nombre d'occurrences de contact en calculant \\
& 100 mots par analyse \\
\hline Les 4 textes les moins bien notés & $\mathbf{3 3}$ occurrences \\
\hline
\end{tabular}

\subsection{Interprétation des résultats}

Cette analyse indique que tous les candidats ont plus ou moins recours à la L1 en écrivant en langue cible. Ceci corrobore les conclusions de van Weijen \& al. (2009), qui, au vu des résultats de la méthode de la pensée à voix haute, attestent que l'emploi de la L1 varie d'un participant à l'autre, mais aussi qu'en écrivant en L2, tous leurs vingt participants utilisent la L1. Les six participants de l'étude de Knutson (2006) utilisent eux aussi la L1 dans la génération des textes, aux niveaux des mots, des syntagmes ou des phrases. La méthode de la pensée à voix haute lui permet en effet de constater que l'anglais L1 a servi de base à ses participants dans la formulation d'une unité de sens, qui est ensuite modifiée et reconfigurée selon les notions connues des participants, concernant les règles d'ordre des mots, la morphologie, et tout ce qui leur paraît spécifique au français langue cible. Dans notre cas, sur 36 copies, une seule, rédigée peut-être par un natif, ne semble contenir aucune occurrence d'influence provenant de la L1.

L'étude indique aussi, comme il est soutenu dans plusieurs recherches (Knutson 2006 ; van Weijen \& al. 2009), que les apprenants plus compétents en écriture sont moins susceptibles d'utiliser leur L1. Selon Knutson, les apprenants possédant moins de ressources en L2 ont constamment dû utiliser la L1 pour générer leur texte, alors que l'apprenant le plus compétent a généré son texte le plus souvent directement en L2. Nous souscrivons à l'avis des auteurs qui tentent d'expliquer ce phénomène en avançant que les apprenants moins compétents en écriture subissent une surcharge cognitive ou une surcharge de la mémoire de travail en rédigeant un texte en L2. Dans ce cas, l'alternance automatique vers la langue la mieux connue permet de traiter l'information en minimisant la charge qui pourrait être alourdie par l'emploi de la langue moins bien connue (Qi 1998). 
En revanche, les apprenants plus doués risquent bien moins de subir cette surcharge cognitive, grâce à leur compétence plus avancée en écriture (van Weijen \& al. 2009).

Ceci pourrait aussi en partie expliquer la tendance que les candidats plus avancés dans leur apprentissage de FLE recourent sensiblement moins à la L1 que les apprenants du niveau Intermédiaire. Ceci est en conformité avec les observations préalables, qu'aux stades plus précoces du développement linguistique, la L1 s'utilise plus largement et se révèle plus nécessaire. Il se dégage, en effet, de l'étude de Qahfarokhi \& Biria (2012), que tant dans le cas des tâches faciles que dans celui des tâches plus exigeantes, les participants de niveau Intermédiaire ont alterné les codes bien plus souvent que les apprenants de niveau Avancé.

La difficulté de la tâche est effectivement un autre facteur qui influe sur la fréquence de l'alternance translinguistique (Qahfarokhi \& Biria 2012). Pour Qi (1998), l'étendue de l'alternance varie entre les tâches présentant différents niveaux d'exigences cognitives. Plus une tâche demandait un haut niveau de connaissance, plus sa participante était susceptible de recourir à sa L1 en tant que stratégie implicite ou explicite de solution des problèmes. Dans notre cas, il n'est pas facile de déterminer pour quelle raison ce sont les textes des candidats ayant reçu les meilleures notes qui contiennent le plus d'occurrences de contact dans les Analyses d'un texte non littéraire. Si on regarde de plus près les types d'occurrences dans cet exercice, on trouve que tant dans les copies les mieux notées que dans les textes ayant obtenu une note inférieure, les occurrences liées à la technique de l'exercice sont bien plus fréquentes que celles qui sont simplement des résultats des carences linguistiques en L2, à un ratio de 7 pour 3 dans les deux catégories de copies. Les occurrences liées à la technique de l'exercice incluent différents phénomènes qui laissent noter l'influence de la L1, comme l'orthographe des mots spécialisés (context, text, paragraph), le choix d'items lexicaux comme phrase pour syntagme, focus pour emphase, topics pour sujets, des approximations comme le vocabulaire s'extend; les phrases dans ce texte sont complicatives, et des occurrences de nature morphosyntaxique comme L'auteur est absent dans le texte parce qu'il n'inclut pas lui-même ; l'auteur du texte utilise nombreux adjectives... ; des mots chargées en valeur... La supériorité numérique d'items appartenant au jargon technique pourrait constituer un indice pour comprendre l'inversion des tendances : les candidats plus compétents pourraient être plus attentifs à la technique et utiliseraient par la suite davantage de termes et de formes spécialisés, étroitement liés à la technique de l'exercice, en le traitant de manière plus approfondie et en écrivant des textes plus longs et plus spécialisés.

L'analyse du corpus révèle aussi que contrairement aux copies étudiées par Caruana (2011), où le maltais et l'anglais étaient les seules langues en contact avec l'italien langue cible, dans les copies de français, le transfert linguistique dérive aussi, et assez souvent, de l'italien. Ceci contribue à confirmer l'italien en tant que troisième langue en importance à Malte, avec l'habileté d'influencer la performance des apprenants d'autres langues étrangères. Nous avons aussi noté l'influence d'autres langues, avec l'apparition, par exemple, de mots espagnols comme l'ingles (Av. Rédaction septembre 4) et coûtumbres (Av. Rédaction mai 12). Caruana indique le maltais comme source particulièrement importante du transfert, surtout au niveau lexical, ce qui s'explique de par les nombreux mots maltais qui s'apparentent à des mots italiens dont ils dérivent. Dans notre cas, il semble à première vue que l'anglais soit le point de départ de la plupart des occurrences de contact. Ceci serait peut-être le cas à cause du statut de 'langue seconde' et de véhicule de l'enseignement dont est doté l'anglais à Malte, ce qui amènerait les apprenants à l'impliquer davantage dans leur travail scolaire. A ce stade, ceci reste pourtant à confirmer puisqu'il faut bien plus de calculs pour déterminer la langue qui est la principale source d'influence sur l'écrit des apprenants maltais de FLE. 
Un autre aspect à propos duquel il est au terme de cette étude encore difficile de se prononcer avec certitude est l'ampleur de l'effet du contexte bilingue ou plurilingue de Malte sur la quantité de cas d'occurrences de contact dans les textes écrits analysés. Caruana (2011 : 370) semble voir un rapport de cause à effet entre les deux («In base a queste considerazioni [la fréquence de l'alternance codique à Malte, dans la communication verbale en classe et au-delà] non è sorprendente riscontrare moltissimi casi di contatto linguistico nella produzione scritta in italiano di apprendenti maltesi »). Nous sommes aussi de l'avis que le comportement d'alternance 'instinctif' des Maltais pourrait contribuer aux proportions dans lesquelles les occurrences figurent dans les textes rédigés en FLE par les apprenants maltais. C'est pourquoi nous nous sommes attardée à décrire ce comportement au début du présent travail. Néanmoins, nous nous garderons de voir dans l'alternance habituelle des Maltais la seule ou la principale cause du contact à l'écrit des apprenants en L2 ; les chercheurs attestent le recours à la L1 dans l'écriture en L2 même dans des pays où la L1 est la langue fortement dominante dans le scénario linguistique et où l'alternance est peu présente dans la communication quotidienne, comme dans les contextes des études de Knutson, 2006, van Weijen \& al. (2009), et Qahfarokhi \& Biria, 2012. L'écriture en L2 paraît être un processus bilingue, indépendamment du caractère principalement monolingue ou multilingue du contexte géographique dans lequel les scripteurs se livrent à la création de leurs textes.

\subsection{Conclusion}

Cette étude de la production écrite en L2 révèle de manière générale que dans le contexte examiné, la fréquence d'items lexicaux portant l'influence de la L1 n'est pas négligeable. Elle tend à devenir plus importante dans les écrits des apprenants moins avancés dans leur parcours d'apprentissage comme dans les textes des apprenants moins compétents en L2. Les occurrences de contact se multiplient aussi lorsque la tâche est plus exigeante.

Alors qu'un nombre de chercheurs interprètent l'alternance avec la L1 dans la rédaction en L2 comme avantageuse pour la qualité du texte final et durant le processus de rédaction, par son rôle d'étayage et de facilitation (Knutson, 2006 ; Qahfarokhi \& Biria, 2012 ; Qi, 1998), les examinateurs maltais sanctionnent négativement l'apparition de termes porteurs de l'influence d'autres langues. Le rapport post-examen rédigé par les examinateurs du niveau Avancé de 2018 explicite que : "Interference with other languages was also a cause of loss of marks, for example, "apreste attencion", "personalement"; the most striking was perhaps "les buses" " (Matsec 2018). En ceci, les examinateurs semblent être du même avis que Gevers (2018), selon qui les langues sont contraintes par les demandes du cadre socioculturel et le mode de communication. Les examens constituent un cadre formel avec des exigences précises, excluant les approximations et les emprunts.

Cette étude présente un nombre de carences méthodologiques, au stade actuel de son progrès. Le corpus contenant 36 textes écrits pourrait être agrandi, afin de permettre une généralisation plus fiable des résultats. Ceci permettrait aussi que les calculs statistiques simples soient vérifiés par des tests formels de signification qui pourraient tester les conclusions tirées ici par simple inférence. L'étude ci-dessus se limite à deux genres d'écriture ; elle pourrait être développée pour inclure par exemple l'exercice du résumé du texte effectué au niveau Intermédiaire.

Le corpus constitué de copies d'examens ne fournit probablement pas le meilleur tableau de la performance des apprenants aux deux niveaux étudiés et pourrait avoir des implications sur la présence de facteurs d'influence provenant de la L1. Pourtant, les 
motivations de ce choix méthodologique peuvent être expliquées par le fait que le système éducatif maltais repose en grande partie sur des examens fréquents, qui excluent d'autres formes d'évaluation. Heureusement, des mesures ont commencé à être prises plutôt récemment pour pallier ce problème. Néanmoins, il reste très important de construire la meilleure compréhension possible de ce qui se passe dans ce contexte et des implications des examens pour le progrès académique et le bien-être des apprenants. Les corpus de copies d'examens sont fiables en ce sens qu'ils assurent que l'apprenant ait travaillé indépendamment. Ils offrent aussi les avantages pratiques d'être relativement faciles à obtenir, sont représentatifs du groupe entier des apprenants et sont anonymes à la source.

Les limitations mentionnées ci-dessus inspirent des projets pour des recherches ultérieures qui viennent s'ajouter à l'intention d'agrandir le corpus et d'y inclure davantage de genres. Les résultats obtenus par les lycéens aux niveaux Intermédiaire et Avancé pourraient être comparés à la qualité des écrits des étudiants universitaires, afin de noter si des tendances de contact différentes peuvent être attestées à un niveau de compétence plus élevé. Les résultats de cette étude pourraient aussi être comparés à des écrits qui ne seraient pas produits sous condition d'examen.

L'expérimentation par tests appuyés sur la méthode de la pensée à voix haute permettrait d'établir avec plus de certitude si les occurrences qui paraissent être des instances de contact linguistique le sont effectivement. La présente étude n'a pu que supposer que les phénomènes observés soient le résultat de l'influence de la L1. La méthode d'enregistrer les réflexions des participants pendant l'écriture a été critiquée sous certains aspects, entre autres à cause du doute que la verbalisation à voix haute par un individu de ses pensées en écrivant puisse effectivement simuler la vraie situation de la rédaction (Zamel, 1983). Cependant, comme van Weijen \& al. (2009), nous croyons que la méthode de la pensée à voix haute peut fournir des données utiles à la recherche sur l'écriture en L2 en permettant d'observer le déroulement d'activités conceptuelles telles que la planification, la génération d'idées, et l'auto-évaluation.

Les analyses des erreurs, comme celle de la présente étude, offrent pourtant deux avantages. Leurs corpus peuvent être plus grands que ceux des méthodes à voix haute, qui, par leur rigueur, imposent souvent qu'on limite le nombre de participants et posent donc problème quant à la possibilité de généraliser les résultats. Les analyses des erreurs, de par leur nature, présentent des données réelles et aident à éviter que l'on base les matériaux pédagogiques sur des prédictions hypothétiques et imprécises; elles permettent par contre de cibler les difficultés réelles des apprenants en développant des matériaux centrés sur leurs besoins en matière de syntaxe, de vocabulaire et d'orthographe (Zamel, 1983). Ce qui se dégage pourtant comme une constante à partir des différents types de méthodes de recherche, c'est la nécessité d'examiner les facteurs qui influencent le comportement linguistique de l'alternance codique et ses effets sur la performance dans les tâches écrites. C'est seulement en obtenant ce genre de compréhension que l'on pourra trancher sur l'utilité d'encourager les apprenants à avoir recours à la L1 en rédigeant un texte en L2. L'étude de la rédaction en L2 montre que pour les apprenants, l'écriture en langue étrangère constitue un processus complexe et nécessairement bilingue.

\section{Références bibliographiques}

Busuttil Bezzina, A.M. (2013). La variation stylistique en maltais. Etude des usages concrets de la langue appuyée sur une approche contrastive des phénomènes variationnels en maltais et en français. Thèse de doctorat inédite, Université Paris Nanterre / Université de Malte. 
Bezzina, A.-M. (2016). Teachers' Understanding of the Use of Languages as a Medium of Instruction in 'French as a Foreign Language' Lessons. Malta Review of Educational Research, 10(2), 277296.

Bezzina, A.-M. (2017). Translanguaging Practices in the Teaching of French as a Foreign Language in Malta. Malta Review of Educational Research, 11(1), 75-95.

Bezzina, A.-M. \& Gauci J. (2018). A Sociolinguistic Perspective of Codeswitching in French as a Foreign Language Class in Malta and its Implications for Learning. In Romanowski P. \& Jedynak M. (éds.), Current Research in Bilingualism and Bilingual Education, Cham: Springer Nature, 55-78.

Camilleri Grima, A. \& Caruana, S. (2016). Interaction and Approximation to the Target Language During Italian Lessons in Malta, Malta Review of Educational Research: Special Issue on Bilingualism in Education in Malta, 10(2), 253-275.

Caruana, S. (2011). "Alavolja il suo padre e la sua mamma non folevanno": fenomeni di contatto nella produzione scritta di italiano L2 degli apprendenti maltesi. Lingue e culture in contatto. Actes du 10e Colloque AItLA, 365-381.

Caruana, S. \& Brincat, L. (2011). Il-Malti Mghaggel: Maltese in computer-mediated chat conversations. In: S. Caruana, R. Fabri \& T. Stolz, (éds.) Variation and Change: The dynamic of Maltese in space, time and society. Berlin: Akademie Verlag, 65-89.

Caruana, S. \& Camilleri-Grima, A. (2014). L'italiano a Malta e la commutazione di codice in contesti didattici. In Lingue in contatto / Contact linguistics, Actes du XLVIe Colloque International d'Etudes de la Società di Linguistica italiana (SLI), R. Bombi \& V. Orioles (éds.), Rome: Bulzoni, 79-96.

Friedlander, A. (1990). Composing in English: Effects of a first language on writing in English. In Kroll, B. (éd.), Second Language Writing, New York: CUP, 109-125.

Gauci, H. \& Camilleri-Grima, A. (2013). Codeswitching as a Tool in Teaching Italian. International Journal of Bilingual Education and Bilingualism, 16(5), 615-631.

Gevers, J. (2018). Translingualism revisited: Language difference and hybridity in L2 writing, Journal of Second Language Writing, 40, 73-83.

Gilyard, K. (2016). The Rhetoric of Translingualism. College English, 78(3), 284-289.

Knutson, E.-M. (2006). Thinking in English, Writing in French. The French Review, 80(1), 88-109.

Lee, J.-W. (2016). Beyond Translingual Writing. College English, 79(2), 174-195.

Qahfarokhi, A.-M. \& Biria, R. (2012). The Impact of Task Difficulty and Language Proficiency on Iranian EFL Learners' Code-switching in Writing, Theory and Practice in Language Studies, 2(3), 572-578.

MATSEC Examinations Board (2019) Examiners' Reports 2019, AM French. Available at: https://www.um.edu.mt/_data/assets/pdf_file/0007/417616/AMFREN.pdf

Qi, D.-S. (1998). An Inquiry into Language-switching in Second Language Composing Processes. The Canadian Modern Language Review, 54(3), 413-435.

Sammut, N. (2017). Analyse des erreurs grammaticales en production écrite : le cas des apprenants maltais de FLE en milieu scolaire bilingue. Mémoire de M. Ed. inédit, Université de Malte.

Seychell, L. (1996). Analyse systématique des erreurs: problèmes et perspectives. Msida : Mireva.

Van Weijen, D. et al. (2009). L1 use during L2 writing: An empirical study of a complex phenomenon, Journal of Second Language Writing, 18, 235-250.

Zamel, V. (1983). The Composing Processes of Advanced ESL Students: Six case studies. TESOL Quarterly, 17(2), 165-187. 